\title{
A Wayfinding Grammar Based on Reference System Transformations
}

\author{
Peter Kiefer, Simon Scheider, Ioannis Giannopoulos, and Paul Weiser \\ Institute of Cartography and Geoinformation \\ ETH Zürich \\ Stefano-Franscini-Platz 5 \\ CH-8093 Zürich, Switzerland, \\ \{pekiefer, sscheider, igiannopoulos, pweiser\}@ethz.ch
}

\begin{abstract}
Wayfinding models can be helpful in describing, understanding, and technologically supporting the processes involved in navigation. However, current models either lack a high degree of formalization, or they are not holistic and perceptually grounded, which impedes their use for cognitive engineering. In this paper, we propose a novel formalism that covers the core wayfinding processes, yet is modular in nature by allowing for open slots for those spatial cognitive processes that are modifiable, or not yet well understood. Our model is based on a formal grammar grounded in spatial reference systems and is both interpretable in terms of observable behavior and executable to allow for empirical testing as well as the simulation of wayfinding.
\end{abstract}

Keywords: wayfinding, navigation, spatial cognitive processes, formal grammar, reference systems

\section{Introduction and Related Work}

Navigation, i.e., the combined endeavor of both locomoting and wayfinding, is an activity most people carry out on a daily basis. While locomotion can be defined as the coordinated movement in the nearby environment in order to avoid obstacles, wayfinding refers to "the planning and decision-making necessary to reach a destination" [32]. Successful wayfinding consists of a wide variety of cognitive processes that can be distributed through time and among individuals (cf. $[44,43,10,13])$, as well as involve the coordination of internalized and externalized spatial knowledge (cf. [30]). In fact, human cognition goes beyond "what is inside our heads alone" by encompassing "the cognitive roles of the social and material world" [12].

Research in spatial cognition has long wondered about the nature of the cognitive processes that make up navigation and wayfinding and attempted to model them. For example, Downs and Stea proposed that wayfinding consists of orientation (Where am I?), route choice (Where should I go?), monitoring (Am I still on track?), and goal recognition (Am I there yet?) [3]. Golledge identified various sub-processes that involve "to determine turn angles, to identify

Kiefer, P., Scheider, S., Giannopoulos, I., and Weiser, P. (2015) A wayfinding grammar based on reference system transformations. In: Conference on Spatial Information Theory XII (COSIT). Springer, 2015, accepted 
segment lengths and directions of movement, to recognize en route and distant landmarks, and to embed the route to be taken in some larger reference frame" [8, p. 7]. Arthur and Passini suggested that wayfinding consists of information processing (perception and interpretation of the environment), decision making (constructing a hierarchical action plan) and decision execution (transformation of a plan into behavior) [2].

In contrast to the aforementioned models that remain descriptive, there also exist formal approaches that are capable of simulating wayfinding based on rule sets that denote condition-action pairs $[9,41,25,24,1]$. However, Golledge [7] criticized that such decision models prescribe a particular way of how decision making takes place that does not match established theories on human cognition. Haken and Portugali [11] suggested to model the interaction of internal and externalized spatial knowledge with neural synergetic networks which involve a variety of feedback loops. Raubal and Worboys [35], in turn, proposed a graph model of possible knowledge and location transitions in an environment that allows representing navigation as a path. However, the model does not address how this graph can be built. A way to summarize route knowledge based on a formal grammar was proposed by Klippel [21]. Yet, while these models are formally specified they only represent some aspects of the wayfinding process. Formal grammars in which rule applications can be spatially constrained were proposed by Schlieder [38] and Kiefer [15,16]. However, these formalisms do not aim specifically at modeling wayfinding, they rather model general intentional behavior.

To conclude this brief review, there is still a lack of formal and operational models that are cognitively plausible and capture the processes of wayfinding from a holistic point of view, without prescribing questionable assumptions regarding decision and search procedures.

In this paper, we propose a novel wayfinding process model based on a formal grammar which can be termed a simulation meta-model. It covers the core wayfinding processes, yet is modular in nature by allowing for open slots to account for those spatial cognitive processes that are modifiable, not well understood, or for which there is no reliable theory yet. These open slots can then later be filled with (ad-hoc) process models, and tested. Our model is interpretable in terms of observable behavior (including perceptual processes and actions) and at the same time executable, such that the wayfinding processes can be simulated. Furthermore, since a major part of the relevant cognitive and perceptual processing consists of interactions with spatial reference systems ${ }^{1}$ our model is grounded in reference systems.

The remainder of this paper is structured as follows. We informally explain our model in Section 2 and introduce the grammar in Section 3, before we illustrate how different kinds of wayfinding scenarios can be simulated (Section 4). We discuss the limitations of our approach and conclude with an outlook in Section 5.

${ }^{1}$ These include: cognitive reference frames, mental survey representations as well as geographic reference systems (cf. [36]). 


\section{Wayfinding in Terms of Reference System Transformations}

In this section we describe the theoretical principles and components of our model. We illustrate how the different wayfinding processes can interact with each other. Note, we do not make any claim on how the processes are actually "implemented" in the human cognitive system. However, we suggest that a central role in generating interaction constraints is played by reference systems and their transformations (cf. [5]).

\subsection{Spatial Reference Systems in Wayfinding}

Spatial reference systems are used to refer to locations across individuals and across time [23]. The way how spatial reference is determined and with respect to which ground phenomena is characteristic to the particular reference system. It affects how and to what extend a location can be transformed from one system into another [37]. Cognitive (internalized) reference frames play a fundamental role in learning and remembering space [39], while spatial coordinate (externalized) systems establish the semantics of maps and other forms of spatial data [23]. Each reference system comes with particular kinds of operations that play an important role in the wayfinding process:

1. Egocentric Reference Systems. An egocentric frame is centered and aligned with the body of a perceiving ego. It can be aligned with the direction into which the eyes look (retinal), or it can be aligned with the head or the body front of a person [14]. In any case, an egocentric frame captures a momentary perceptual array ${ }^{2}$ of the ego, with objects and locations perceived in a certain angle and distance from the self (self-to-object). Objects can be both places and bodies with surfaces [14]. Egocentric frames roughly correspond to Vista space [33]. They are closely connected to the perceptual array and thus to direct experience, and are kept primarily in short-term or working memory. They take an important role in motor-control, as well as in projecting locomotion into the perceived environment, and are probably located in the brain's parietal cortex [14]. Their role in wayfinding is that they provide input for self-localization (where am I?) and are output of path-localization (where do I need to go?), both of which form major parts of the required attentional effort.

2. Allocentric Reference Systems and Route Knowledge. Allocentric systems encode locations relative to other objects (object-to-object). Humans can easily transform egocentric locations into allocentric ones (and vice versa) by taking egocentric locations with respect to perceivable ground objects and orientations $[14,39,5]$. For this reason, allocentric systems are able to render locations inter-subjective (i.e., they can be shared among others) and

\footnotetext{
${ }^{2}$ Note that we use this term in an intermodal sense, i.e., not restricted to vision and thus integrating different modalities of perception.
} 
independent of a point of view or movement. They give a particular fixed meaning to qualitative spatial locations [5] (e.g., "in front of"), which are taken with respect to ground objects and some perceivable orientation, and are termed relation templates (cf. [27]). Allocentric reference systems constitute the meaning of large parts of human spatial language [26], as well as spatial memory [39]. They may constitute what Montello calls environmental space, i.e., space that needs to be apprehended through locomotion [33]. We assume that wayfinding knowledge is largely encoded in an allocentric form, more particularly in terms of short term and long-term route memory consisting of sequences of actions and allocentric locations with landmarks as ground objects [31]. For example, we may remember our way to work in terms of the sequence: turn right in front of the church, turn right at the bank, then enter the parking lot. Their role in wayfinding is that they represent route instructions which implement plans based on spatial memory.

3. Survey Reference Systems. While allocentric (cognitive) reference systems already constitute a kind of inter-subjective knowledge, spatial reference remains uncertain when ground objects are not in view or have never been experienced. This renders them unsuitable for survey planning. In the wayfinding process, survey knowledge is indispensable whenever the way extends beyond any location that is describable relative to known ground objects. For this reason, people have learned to use reference systems that represent the geometric configuration of unknown objects and locations [31]. Their role in wayfinding is therefore to support the construction of possible ways to go, i.e., the planning of wayfinding and its simulation in case route memory fails. Survey reference systems are grounded relative to the earth's surface or other ground phenomena that remain in view. They roughly correspond to Montello's geographic space which needs to be "learned via symbolic representations" [33]. One example is a geographic reference system on which geographic maps are based. Their cognitive counterparts are mental representations of survey knowledge ("cognitive maps") which are bird-eye views kept in long-term memory, constructed by cumulative spatial experience or by memorizing geographic maps, and which allow for perspective taking and making spatial inferences (cf. $[42,31])$. Transformations of survey locations to allocentric or egocentric systems are only possible when ground objects and orientations can be mapped. To what extent cognitive maps resemble cartographic maps and whether this analogy is rather a metaphor is debatable [20]. However, it seems fair to assume that some kind of survey knowledge (either internalized or externalized) is necessary for the purpose of wayfinding.

\subsection{Wayfinding Processes and Their Dependencies}

In this section we informally discuss reference system transformations and other cognitive processes that occur during wayfinding. Figure 1 illustrates our model 


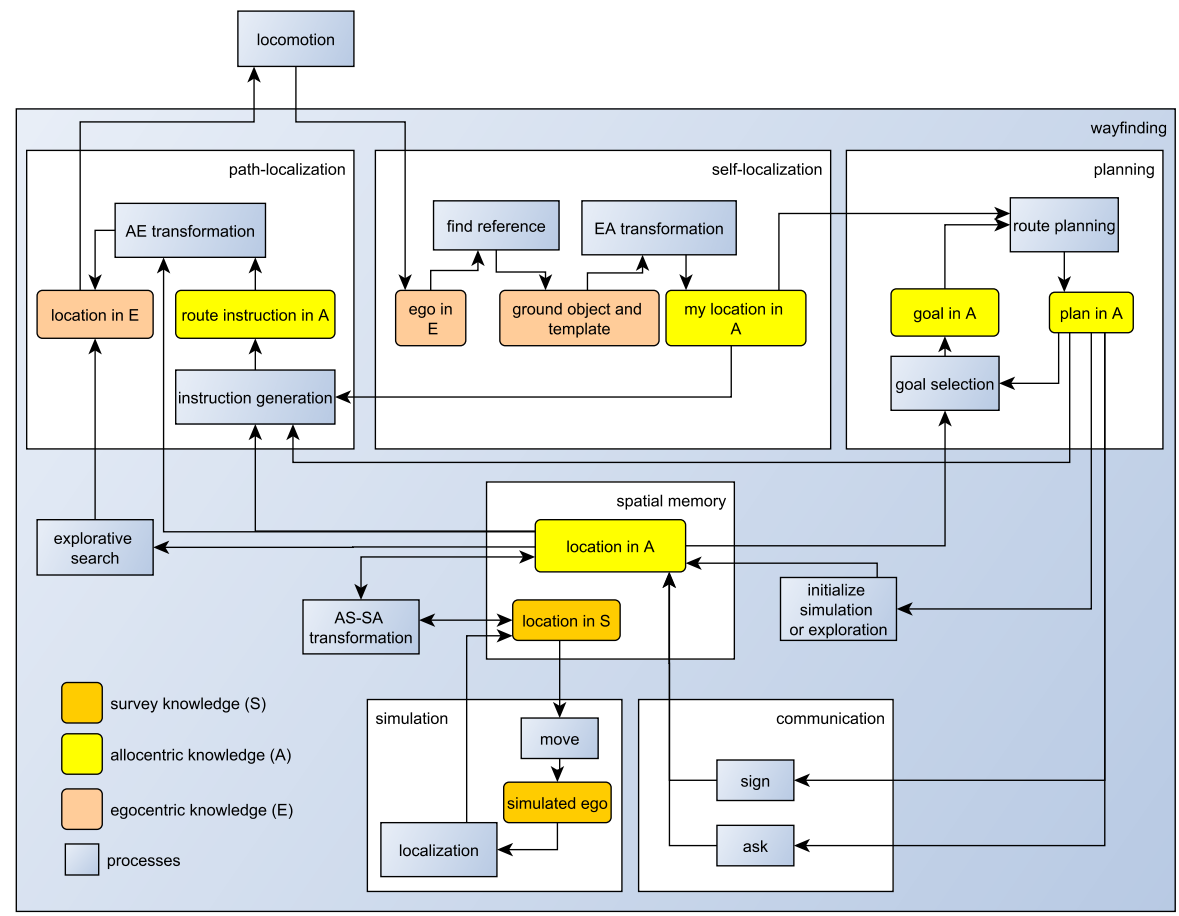

Fig. 1: Modeling the wayfinding process based on reference system transformations.

as a transition graph on different kinds of spatial knowledge and the processes connecting them.

We describe our model using the example of Susi who is trying to find her way to some destination. Susi starts with her egocentric field of view ("ego in E", Fig. 1). Her first task is to figure out where she is, i.e., she needs to localize herself w.r.t. some allocentric system (cf. "orientation" [3]; Fig. 1: box "self-localization" ). That is, she needs to find a spatial referent - a suitable ground object and relation template - and transform her position into an allocentric location. Then Susi starts planning a route by selecting a goal (Fig. 1: box "planning") and by initializing a plan - being simply an intention to go there - which can recursively be divided into sub-plans by selecting subgoals from memory (cf. "route choice" [3] and "decision making" [2]).

Plans can be implemented in several ways. If Susi already knows from past experience how to turn the plan into an instruction, she will be able to retrieve this instruction from memory in terms of route knowledge, e.g., "turn left in front of the church" (Fig. 1: box "path-localization"). If, however, spatial memory does not allow to generate an instruction, she will have several possibilities: For example, she can start an explorative search. In this case, Susi will just "follow 
her nose" in her egocentric system until she reaches a location that is familiar to her (from where she can again start planning), or until she recognizes the goal location. In addition, Susi can ask other people to acquire route knowledge, consult some form of signage (Fig. 1: box "communication"), or search for a route on a map. For instance, a sign pointing into a certain direction describes an allocentric location relative to that sign ('location in A') which then needs to be transformed into an egocentric location ('location in E') through an 'AE transformation'.

Searching for a route on a map is specifically interesting in terms of reference system transformations because then survey knowledge becomes relevant. In our model, we describe map-based wayfinding as navigation simulation on a survey reference system (Fig. 1: box "simulation"). Susi performs a search on the survey map, moving her attention (and/or possibly her finger) to the goal and memorizes this as a sequence of imagined actions and allocentric locations. We assume that a similar simulation can be performed on a mental representation of survey knowledge (mental map). The output of a simulation is the required (sequence of) instruction(s), thus yielding route knowledge.

In case an instruction can be transformed into her egocentric system (Fig. 1: box "AE transformation"), Susi can start locomoting, which will populate her field of view with new objects that may trigger new transformations from egocentric systems to allocentric ones. Susi may continue like this as long as instructions can continuously be generated from memory and as long as locations can be transformed. If available memory runs out, or if an instruction cannot be interpreted w.r.t. perception, she will need to start the whole process again (i.e., start with self-localization based on "ego in E").

\section{$3 \quad$ Wayfinding Grammar}

We model wayfinding as the process of planning and constructing wayfinding instructions, and translating them from survey or allocentric locations into egocentric reference systems (i.e., locations in the field of view of the ego). Instructions can be generated by experience, survey simulation, or communication (see also Fig. 1).

For this purpose, we use a formal grammar, i.e., a set of rewriting rules that transform a non-terminal start symbol into a string of terminal symbols. Using a formal grammar, wayfinding can be represented as a sequence of rule applications generating terminals which stand for behaviors, such as locomotion or visual search. Thus, a wayfinding process can be simulated in terms of the rewriting process. Furthermore, similar to the syntax of a language, the grammar defines allowable sequences of behaviors ("well-formed formulae"), so that a parser can check whether a given string of measured behaviors can denote a wayfinding process.

We first define the grammar in terms of symbol sets and rules, before explaining how the elements of the grammar interact. The scenarios in Section 4 illustrate how the grammar can be used. 
Definition 1. (Locations) Let $\mathbf{S}$ denote a finite set of objects in a survey reference system, $\mathbf{A}$ a finite set of allocentric systems, and $\mathbf{E}$ a finite set of objects in an ego-centric reference system. We define $\mathbf{L}^{\mathbf{S}} \subseteq \mathbf{S} \times \mathbf{R e l}, \mathbf{L}^{\mathbf{A}} \subseteq \mathbf{A} \times \mathbf{R e l}$, and $\mathbf{L}^{\mathbf{E}} \subseteq \mathbf{E} \times \mathbf{R e l}$ as the sets of survey, allocentric, and egocentric locations w.r.t a (finite) set of spatial relation templates $\mathbf{R e l}$. The set of all locations is then defined as $\mathbf{L}=\mathbf{L}^{\mathbf{S}} \cup \mathbf{L}^{\mathbf{A}} \cup \mathbf{L}^{\mathbf{E}}$.

Spatial relation templates $\mathrm{rel}_{i} \in \mathbf{R e l}$ can be used to describe a location relative to a reference object, such as $L_{\text {frontOfChurch }}^{A}=\left(A_{\text {church }}\right.$, rel $\left.l_{\text {inFrontOf }}\right)$, with $A_{\text {church }}$ $\in \mathbf{A}, L_{\text {frontOfChurch }}^{A} \in \mathbf{L}^{\mathbf{A}}$.

Definition 2. (WAYFINDING GRAmmaR) A wayfinding grammar is a contextfree production system $\left(\mathbf{B}, \mathbf{N}, \mathbf{R}, E_{\text {ego }}, L_{g}^{A}, A c c\right)$ with

- $\mathbf{B}$ denoting a set of wayfinding behaviors (the terminals, see Definition 3)

- $\mathbf{N}=\left\{P\left[L_{i}^{A}\right]\left[L_{j}^{A}\right], I\left[L_{k}^{A}\right]\left[L_{l}^{A}\right]\right\} \cup \mathbf{L} \cup \mathbf{E} \cup \mathbf{S}$ defining the non-terminals. The plan

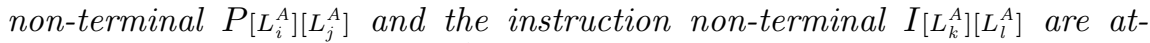
tributed with locations from $\mathbf{L}^{\mathbf{A}}$ (the 'from' and the 'to' location).

$-\mathbf{R}$, a set of production rules from $\mathbf{N}$ to $(\mathbf{N} \cup \mathbf{B})^{+}$(see Table 1)

$-E_{\text {ego }} \in \mathbf{E}$ is the start symbol denoting the perceiving ego

$-L_{g}^{A} \in \mathbf{L}^{\mathbf{A}}$ denoting the allocentric goal location of the wayfinding process.

- Acc $\subseteq \mathbf{L}^{\mathbf{A}} \times \mathbf{L}^{\mathbf{A}} \times \mathbf{H}$ represents a wayfinder's route knowledge as a graph over $\mathbf{L}^{\mathbf{A}}$ with edge labels from the set of headings $\mathbf{H}$, which denote turn directions such as "turn left" or "turn north". $\left(L_{i}^{A}, L_{j}^{A}, h_{k}\right) \in$ Acc means "the wayfinder knows that $L_{j}^{A}$ is directly accessible from $L_{i}^{A}$ in direction $h_{k}$ ".

Definition 3. (WAYFINDING BeHAVIORs) The set of wayfinding behaviors B contains the following elements goto $\left[L_{i}^{E}\right] \quad$ The wayfinder locomotes to $L_{i}^{E}$.

$\mathbf{H} \quad$ The wayfinder changes heading to $h_{i} \in \mathbf{H}$.

$s_{A E}\left[L_{i}^{A}\right] \quad$ Search for allocentric location $L_{i}^{A}$ in egocentric system.

$s_{E A}\left[L_{i}^{E}\right] \quad$ Search for egocentric location $L_{i}^{E}$ in allocentric system.

$s_{A S}\left[L_{i}^{A}\right] \quad$ Search for allocentric location $L_{i}^{A}$ in survey system.

$s_{S A}\left[L_{i}^{S}\right] \quad$ Search for survey location $L_{i}^{S}$ in allocentric system.

$s_{\text {exp }} \quad$ Search during explorative wayfinding.

$s_{\text {sign }} \quad$ Search on signage.

$s_{\text {refE }} \quad$ Search for referent object in egocentric system.

$s_{\text {refS }} \quad$ Search for referent object in survey system.

sim The wayfinder simulates navigation in a survey system.

sim $_{\text {end }} \quad$ The wayfinder stops survey simulation.

$\operatorname{ask}\left[L_{s}^{A}\right]\left[L_{d}^{A}\right] \quad$ The wayfinder requests instructions to go from $L_{s}^{A}$ to $L_{d}^{A}$

We use an attributed context-free grammar [22] (see Definition 2). Non-terminals for plan and instruction are attributed with allocentric locations denoting the "from" and "to" locations. This is used to constrain the application of rules and it effectively turns grammar rules into meta-rules: allowable rule sets can be derived by substituting variables with elements from $\mathbf{L}$ (cf. Table 1). 
Table 1: Meta rules of the wayfinding grammar.

\begin{tabular}{|c|c|c|}
\hline Production Meta Rule & Triggers & Description \\
\hline \multicolumn{3}{|l|}{ Planning } \\
\hline $\begin{array}{l}L_{i}^{A} \rightarrow P\left[L_{i}^{A}\right]\left[L_{g}^{A}\right] \\
\text { with } L_{g}^{A}=\text { goal from grammar definition, } \\
L_{i}^{A} \neq L_{g}^{A} ; \text { rule applied at most once }\end{array}$ & $\begin{array}{l}f_{\text {Sub-plan }}, \\
f_{\text {Impl }}\end{array}$ & (1) plan initialization \\
\hline $\begin{array}{l}P\left[L_{s}^{A}\right]\left[L_{d}^{A}\right] \rightarrow P\left[L_{s}^{A}\right]\left[L_{i}^{A}\right] P\left[L_{i}^{A}\right]\left[L_{d}^{A}\right] \\
\text { with } L_{s}^{A} \neq L_{d}^{A}, L_{s}^{A} \neq L_{i}^{A}, L_{i}^{A} \neq L_{d}^{A}\end{array}$ & $\begin{array}{l}f_{\text {Sub-plan }} \\
f_{\text {Impl }}\end{array}$ & (2) sub-planning \\
\hline $\begin{array}{l}P\left[L_{s}^{A}\right]\left[L_{d}^{A}\right] \rightarrow I\left[L_{s}^{A}\right]\left[L_{d}^{A}\right] \\
\text { with } L_{s}^{A} \neq L_{d}^{A}\end{array}$ & $\begin{array}{l}f_{\text {Instr }} \\
f_{\text {Re-plan }}\end{array}$ & $\begin{array}{l}\text { (3) instruction substitution } \\
\text { from memory }\end{array}$ \\
\hline $\begin{array}{l}I\left[L_{s}^{A}\right]\left[L_{d}^{A}\right] \rightarrow P\left[L_{s}^{A}\right]\left[L_{d}^{A}\right] \\
\text { with } L_{s}^{A} \neq L_{d}^{A}\end{array}$ & $\begin{array}{l}f_{\text {Sub-plan }} \\
f_{\text {Impl }}\end{array}$ & (4) re-planning \\
\hline $\begin{array}{l}I\left[L_{s}^{A}\right]\left[L_{d}^{A}\right] \rightarrow h_{i} L_{d}^{A} \\
\text { with }\left(L_{s}^{A}, L_{d}^{A}, h_{i}\right) \in A c c, L_{s}^{A} \neq L_{d}^{A}\end{array}$ & & (5.1) instruction generation \\
\hline $\begin{array}{l}I\left[L_{s}^{A}\right]\left[L_{d}^{A}\right] \rightarrow h_{i} L_{z}^{A} I\left[L_{z}^{A}\right]\left[L_{d}^{A}\right] \\
\text { with }\left(L_{s}^{A}, L_{z}^{A}, h_{i}\right) \in A c c \\
L_{s}^{A} \neq L_{d}^{A}, L_{s}^{A} \neq L_{z}^{A}, L_{z}^{A} \neq L_{d}^{A} \\
\end{array}$ & $\begin{array}{l}f_{\text {Instr }} \\
f_{\text {Re-plan }}\end{array}$ & (5.2) instruction generation \\
\hline \multicolumn{3}{|l|}{ TRANSFORMATION } \\
\hline$L_{i}^{A} \rightarrow s_{A E}\left[L_{i}^{A}\right] L_{j}^{E}$ & & (6) egocentric matching \\
\hline$L_{i}^{E} \rightarrow s_{E A}\left[L_{i}^{E}\right] L_{j}^{A}$ & & (7) reverse egoc. matching \\
\hline$L_{i}^{A} \rightarrow s_{A S}\left[L_{i}^{A}\right] L_{j}^{S}$ & & (8) survey-matching \\
\hline$L_{i}^{S} \rightarrow s_{S A}\left[L_{i}^{S}\right] L_{j}^{A}$ & & (9) reverse survey-matching \\
\hline$E_{i} \rightarrow s_{\text {refE }} L_{j}^{E}$ & & (10) egocentric localization \\
\hline$S_{i} \rightarrow s_{\text {refS }} L_{j}^{S}$ & $f_{\text {RemS }}$ & (11) survey localization \\
\hline \multicolumn{3}{|l|}{ LOCOMOTION } \\
\hline$L_{i}^{E} \rightarrow \operatorname{goto}\left[L_{i}^{E}\right]$ & $f_{\text {Perc }}, f_{\text {RemE }}$ & $\begin{array}{l}\text { (12) locomotion of the ego to } \\
\text { the new location } L_{i}^{E}\end{array}$ \\
\hline$P\left[L_{s}^{A}\right]\left[L_{d}^{A}\right] \rightarrow L_{d}^{A}$ & $f_{E x p}$ & (13) initialize exploration \\
\hline $\begin{array}{l}L_{i}^{A} \rightarrow h_{i} s_{\exp } L_{j}^{E} L_{i}^{A} \\
\text { with }\left(L_{i}^{A} \rightarrow s_{A E}\left[L_{i}^{A}\right] L_{j}^{E}\right) \notin \mathbf{R}\end{array}$ & $f_{E x p}$ & $\begin{array}{l}\text { (14) explorative search for } \\
L_{i}^{A}\end{array}$ \\
\hline \multicolumn{3}{|l|}{ COMmunication } \\
\hline$P\left[L_{s}^{A}\right]\left[L_{d}^{A}\right] \rightarrow s_{\text {sign }} I\left[L_{s}^{A}\right]\left[L_{d}^{A}\right]$ & $f_{\text {RemC }}$ & (15) use signage \\
\hline$P\left[L_{s}^{A}\right]\left[L_{d}^{A}\right] \rightarrow \operatorname{ask}\left[L_{s}^{A}\right]\left[L_{d}^{A}\right] I\left[L_{s}^{A}\right]\left[L_{d}^{A}\right]$ & $f_{\text {RemC }}$ & (16) ask for instructions \\
\hline \multicolumn{3}{|l|}{ Simulation } \\
\hline $\begin{array}{l}P\left[L_{s}^{A}\right]\left[L_{d}^{A}\right] \rightarrow L_{s}^{A} I\left[L_{s}^{A}\right]\left[L_{d}^{A}\right] \\
\text { with } L_{s}^{A} \neq L_{d}^{A}\end{array}$ & $f_{\text {Sim }}$ & $\begin{array}{l}\text { (17) instruction substitution } \\
\text { with simulation }\end{array}$ \\
\hline$L_{i}^{S} \rightarrow \operatorname{sim} h_{i} S_{\text {egosim }}$ & replace: (11) & (18) simulated locomotion \\
\hline$L_{i}^{A} \rightarrow \operatorname{sim}_{\text {end }}$ & remove: (19) & (19) end of simulation \\
\hline
\end{tabular}


Table 2: Kinds of procedures for wayfinding production. Numbers in brackets refer to rules in Table 1

\begin{tabular}{|c|c|c|c|}
\hline Procedure & Purpose & Result & Triggered on \\
\hline \multicolumn{4}{|c|}{ PRODUCTION PROCEDURES } \\
\hline$f_{\text {Init }}$ & $\begin{array}{l}\text { generate start symbol, static } \\
\text { rules and select goal }\end{array}$ & $\begin{array}{l}\text { generate } E_{\text {ego }}(1)(8) \\
(9)(11), \text { set } L_{g}^{A}\end{array}$ & start \\
\hline \multicolumn{4}{|c|}{ PLAN UPDATING } \\
\hline$f_{\text {Sub-plan }}$ & $\begin{array}{l}\text { Select a sub-goal for a given } \\
\text { plan from internal memory }\end{array}$ & generate $(2)$ & (1) and (4) \\
\hline$f_{\text {Impl }}$ & $\begin{array}{l}\text { Generate rules that implement } \\
\text { a plan }\end{array}$ & $\begin{array}{l}\text { generate (3) (13) (15) } \\
(16)(17)\end{array}$ & (1) $(2)(4)$ \\
\hline$f_{\text {Instr }}$ & $\begin{array}{l}\text { Select edge from route knowl- } \\
\text { edge (Acc) to build instruction }\end{array}$ & $\begin{array}{l}\text { activate }(6) \text { deac- } \\
\text { tivate (8) generate } \\
(5.1)(5.2)\end{array}$ & $\begin{array}{l}(3)(5.2)(15) \\
(16)(17)\end{array}$ \\
\hline$f_{\text {Re-plan }}$ & Offer a re-planning possibility & generate (4) & $\begin{array}{l}(3)(5.2)(15) \\
(16)(17)\end{array}$ \\
\hline \multicolumn{4}{|c|}{ EGOCENTRIC UPDATING } \\
\hline$f_{\text {Perc }}$ & $\begin{array}{l}\text { Replace egocentric locations } \\
\text { and their transformations }\end{array}$ & $\begin{array}{l}\text { update (6) (7) (10) } \\
(12)\end{array}$ & (12) and start \\
\hline$f_{E x p}$ & $\begin{array}{l}\text { Select egocentric location for ex- } \\
\text { plorative search }\end{array}$ & generate $(14)$ & $(13)$ \\
\hline \multicolumn{4}{|c|}{ SURVEY UPDATING } \\
\hline$f_{\text {Sim }}$ & $\begin{array}{l}\text { switch to simulation mode and } \\
\text { simulate }\end{array}$ & $\begin{array}{l}\text { activate }(8) \text { deac- } \\
\text { tivate }(6) \text { generate } \\
(19)(18)\end{array}$ & $(17)$ \\
\hline \multicolumn{4}{|c|}{ MEMORY UPDATING } \\
\hline$f_{\text {RemE }}$ & $\begin{array}{l}\text { store locomotion experience in } \\
\text { memory }\end{array}$ & update edges in Acc & $(12)$ \\
\hline$f_{\text {RemS }}$ & $\begin{array}{l}\text { store simulation experience in } \\
\text { memory }\end{array}$ & update edges in Acc & (11) \\
\hline$f_{\text {Rem } C}$ & $\begin{array}{l}\text { store communicated knowledge } \\
\text { in memory }\end{array}$ & update edges in Acc & $(15)(16)$ \\
\hline
\end{tabular}




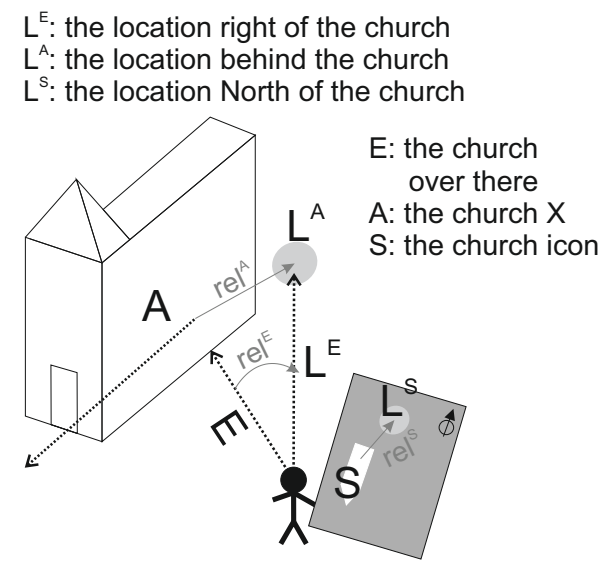

(a) Reference systems in wayfinding. The same location may be egocentric "right of", allocentric "behind", and "North of" the church on the survey level.

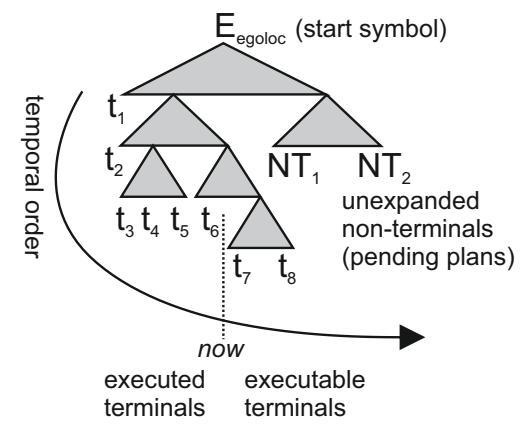

(b) Production tree of a wayfinding grammar. Non-terminals are substituted (topdown direction) and terminals are executed (left-right direction).

Fig. 2

Rules are successively applied to non-expanded non-terminals in order to expand them, generating a production tree, similar to normal context-free grammars (cf. Fig. 2b). A left-to-right and depth-first traversal on these trees determines the temporal order of the resulting terminal sequence of wayfinding behaviors. The wayfinder can execute a terminal (e.g., start locomoting) as soon as there is no unexpanded non-terminal or non-executed terminal left of it. Example production trees for our grammar are presented in Section 4.

Note that the modeled wayfinding behavior (the terminals) may or may not be observable in a specific case, depending on the sensor technology available. If certain terminals are not measurable in a given scenario they can simply be removed from the grammar. For instance, terminals goto $\left[L_{i}^{E}\right]$ and $h_{i}$ require a position and directional sensor respectively, while the search behaviors $s_{x}$ can be derived from measuring eye movements with eye tracking [19]. Terminals denoting search for specific objects are attributed with the object for which is searched. It is known that eye movements can be aggregated and classified to certain types of visual search [18], and we assume that the listed types of search can be distinguished accordingly. During visual search on a map-based survey system the eye tracking data need to be matched with features on the map [17] to detect which objects exactly are looked at.

Our grammar is not static since the sets of egocentric non-terminals $\mathbf{E}$ and locations $\mathbf{L}^{\mathbf{E}}$, the rule set $\mathbf{R}$, and the accessibility graph $A c c$ representing route knowledge can be changed during grammar execution. This is to account for the fact that wayfinding knowledge and the perceptual array change as ego finds its way through an environment. We model the dynamic aspects by using updating 
procedures (See Table 2), which are triggered as indicated in column 2 of Table 1. Updating procedures generate new rules and activate or deactivate others depending on the state of the wayfinding process. They remain external to our grammar and represent open slots in our model, thus need to be filled with realistic sub-models, perceptual processes or measurement procedures.

Furthermore, since the wayfinding process can fail, grammar execution can fail, too. For example, if an instruction is not up-to-date, it may not be transformable into egocentric locations because its description cannot be matched with perception. In terms of our grammar, this simply means that the instruction cannot be fully expanded to terminals. In this case, the old production tree is discarded and the production process simply starts anew with self-localization.

In the following, we explain the semantic interpretation of the grammar and the updating processes. Numbers in parentheses refer to the corresponding rules in Table 1.

\subsection{Self Localization}

The start symbol of our grammar is the ego $E_{\text {ego }}$. The first thing ego needs to do is to self-localize itself w.r.t to an object in its field of view. That is, an egocentric ground object from $\mathbf{E}$ (objects in ego ref. system) and a relation template from Rel must be selected which specify ego's location. For instance, $L_{\text {egoloc }}^{E}$ $=\left(E_{\text {pub }}, r e l_{\text {outside }}\right)$ would describe an egocentric location "outside of the pub from the perspective of ego". Rule (10) models this self-localization. It involves a search for a referent object and a relational template in the egocentric system (terminal $s_{\text {refE }}$ ). There is usually more than one way of describing the egocentric location, so the wayfinder can choose from several rules of type (10). These rules are created for $E_{\text {ego }}$ by procedures $f_{\text {Init }}$ and $f_{\text {Perc }}$ (See Table 2). Once $L_{\text {egoloc }}^{E}$ has been determined it can be used to transform the location of ego to an allocentric location which provides the input for planning. Rule (7) expresses that an egocentric location from $\mathbf{L}^{\mathbf{E}}$ can be transformed into an allocentric representation from $\mathbf{L}^{\mathbf{A}}$ with a search (terminal $s_{E A}\left[L_{i}^{E}\right]$ ), taking into account a perceivable orientation and the geometry of the ground object. For instance, the location $L_{\text {egoloc }}^{E}$ from the example above could be transformed into the allocentric location "in front of the pub" (In a reference system grounded in the pub and looking into the direction of its entrance): $L_{\text {egoloc }}^{A}=\left(A_{\text {pub }}\right.$, rel $\left.l_{\text {infront }}\right)$.

\section{$3.2 \quad$ Planning}

The wayfinding process often involves planning on several levels of abstraction [34]. We start the process with rule (1) which initializes the plan to go from the current location to the goal location $L_{g}^{A}$. Sub-planning with intermediate locations is achieved by (potentially multiple) applications of rule (2). The choice and order of applying planning rules determines the wayfinder's planning strategy which can be modeled by procedures $f_{\text {Sub-plan }}$ and $f_{\text {Impl }}$ (See Table 2) but are out of the scope of this paper. 
In our grammar, there are four ways of continuing with a given plan. First, one can implement the plan by using an instruction from route memory (rule (3)). Second, one can simulate wayfinding in a survey reference system (rules (17) to (19)). Third, one can ask some other agent (rule (16)) or consult signage (rule (15)) in order to obtain an instruction. Finally, one can do explorative search for the goal just by following one's nose (rules (13) and (14)). We will explain the latter three possibilities in the following subsections.

Using rule (3), plans can be substituted by instructions which are detailed sequences of actions and accessible allocentric locations constructed based on route knowledge stored in the accessibility graph Acc. The process of selecting and adding information to an instruction can be modeled by $f_{\text {Instr }}$ (See Table 2). An instruction is given by a heading information $h_{i}$ and an allocentric location $L_{i}^{A}$ (rules (5.1) and (5.2)): the wayfinder turns into a certain direction where $L_{i}^{A}$ is supposed to be found. If $L_{i}^{A}$ is not yet the destination of the instruction sequence (rule (5.2)) further instructions are necessary.

Only if the left-most branch of the resulting production tree has been expanded with an instruction, the ego can start transforming $L_{i}^{A}$ from this instruction into an egocentric location which enables locomotion. This transformation is described by rule (6) which requires a search (terminal $\left.s_{A E}\left[L_{i}^{A}\right]\right)$. That is, the allocentric location $L_{i}^{A}$ (e.g., "behind the church") is turned into an egocentric location accessible from the wayfinder's current position (e.g., "right of the church") (See Figure 2a). Note, if $L_{i}^{A}$ could not be successfully interpreted (i.e., matched onto the environment), one can either do an explorative search for $L_{i}^{A}$ by rule (14), or abort and start anew ${ }^{3}$.

\subsection{Locomotion}

Locomotion moves the ego through space and, in this way, continuously generates new fields of view. In our formalism, this means updating the set of egocentric (perceived) objects $\mathbf{E}$ by procedure $f_{\text {Perc }}$ (See Table 2), i.e., new egocentric objects enter and old objects leave the field of view, similar to the flow in a perceptual array. Corresponding egocentric locations $\mathbf{L}^{\mathbf{E}}$ and transformation rules need to be updated as well. That is, transformation rules with egocentric locations as rule head or body that are out of view will be deleted and transformation rules with novel egocentric objects enter the rule set. $f_{P e r c}$ is triggered every time a new physical locomotion to a given egocentric location is performed by generating goto $\left[L_{i}^{E}\right]$ using rule (12).

Locomotion can be triggered in wayfinding by instruction as well as in explorative search (following one's nose). Rule (13) models the intialization of explorative search from a plan that cannot be resolved to sub-plans (with rule (2)) or substituted with an instruction (with rule (3)). The wayfinder switches from a structured 'planning mode' to an 'explorative mode' (triggering $f_{E x p}$ in Table 2 ). Exploration is then continued by applying rule (14) and (12) in an iterative

\footnotetext{
${ }^{3}$ Note that survey simulation was deactivated with rule (8) by $f_{\text {Instr }}$ in Table 2.
} 
fashion in order to move ego in her field of view without instruction (see example in section 4.2), until the plan destination is reached by chance, i.e., until the allocentric destination on the right hand side of (14) can be transformed to the egocentric system with rule (6).

The rules described so far (rules (1-7), (10), (12-14)) are sufficient for modeling the wayfinding process as a sequence of multi-level planning down to the instruction level or as explorative search, followed by transformations finally leading to changes in heading and locomotion. We have not described, however, the manipulation of (external and internal) spatial memory, but simply assumed that route knowledge is always given. In the following, we describe how new knowledge can be added.

\subsection{Generating Route Knowledge}

In finding our way in an environment, we essentially need to know that we can access a location from another one "directly". If we know that being at $L_{a}^{A}$ implies that we can view and access another location $L_{b}^{A}$ by heading in direction $h_{d i r}$, then we know how to get to $L_{b}^{A}$ once we are at $L_{a}^{A}$ (and so on), even without planning and without physically being at $L_{a}^{A}$.

We model this knowledge with the accessibility graph Acc (see Definition 2). The graph may contain edges at the beginning of wayfinding process if the wayfinder has previous knowledge about an area. The graph can be updated during wayfinding in two ways: either based on egocentric experience, or based on movement simulation in a survey reference system ${ }^{4}$.

In egocentric experience, if we have successfully moved from where we are now to an egocentric location we may add an edge between corresponding allocentric locations to the accessibility graph. That is, the procedure $f_{R e m E}$ in Table 2, which is triggered after application of rule (12), takes the current and the previous goto[ $\left[L_{i}^{E}\right]$ terminals (based on an inverse traversal of the production tree), retraces them to back to allocentric locations $L_{\text {current }}^{A}$ and $L_{\text {previous }}^{A}$, determines the last direction $h_{i}$, and creates an according edge in Acc.

\subsection{Movement Simulation in Survey Reference System}

The second way of adding knowledge to Acc is provided by movement simulation in a survey system (see rules (17)-(19) in Table 1). Note that we make no further assumptions about the nature of these reference systems, e.g., as to whether they are externalized maps or cognitive systems. In fact, they can denote one or the other.

For example, if the wayfinder cannot directly substitute a plan to go from $L_{s}^{A}$ to $L_{d}^{A}$ with an instruction because route knowledge is missing, she may decide to simulate a path by moving her finger on a physical paper map. This process is started by rule (17), which creates an $L_{s}^{A}$ and triggers $f_{\text {Sim }}$ in Table 2 switching

\footnotetext{
${ }^{4}$ In principle, route knowledge may also be removed (forgotten) or overwritten by new experience or simulations.
} 
the grammar to simulation mode, meaning that allocentric locations are now mappable only to survey locations (rule (8) activated), and not to egocentric ones (rule (6) deactivated), and the planning destination is set as simulation goal by adding a corresponding rule (19). $L_{s}^{A}$ can then be mapped to a survey location $L_{s}^{S}$ by rule (8) and becomes the start location for simulation. Next, the simulated ego is moved by rule (18), yielding a new survey position $S_{\text {egosim }}$ (the "finger" is moved on the map). By iterative applications of (18) and (11) a route sequence is recorded until the simulation reaches the destination, leading to an application of rule (19). The simulation terminals ( $\mathrm{sim}$ and $\operatorname{sim}_{\text {end }}$ ) appearing during movement simulation could, for instance, be measured by eye or hand movements. An example for simulation in a survey reference system is presented in section 4.3 .

The main purpose of the simulation process consists in creating new edges in $A c c$, similar to the edge updating by ego-centric movement: if we are able to successfully simulate a path of the simulated ego to a location $L_{j}^{S}$ on a geographic map, and if there exist allocentric transformation rules for these locations, then we can add a corresponding edge to the accessibility graph (procedure $f_{\text {RemS }}$ in Table 2). That is, after application of rule (11), the right-hand location $L_{j}^{S}$ and its grandparent node $L_{i}^{S}$ (see production tree in Fig. 6) are determined. By looking up reverse survey-matching rules $(9)$ for $L_{i}^{S}$ and $L_{j}^{S}$, according allocentric locations $L_{i}^{A}$ and $L_{j}^{A}$ are determined. These are combined with $h_{i}$ (the child node of $L_{i}^{S}$ ) and added to Acc.

\subsection{Obtaining Instructions From Signage or by Asking}

As an alternative to simulation, wayfinders frequently use signage or other communication acts to obtain instructions. In our grammar we represent this with two rules:

Rule (15) models a search on a sign (terminal $s_{s i g n}$ ) which triggers $f_{\operatorname{Rem} C}$ in Table 2 leading to an updated accessibility graph and a new instruction. This instruction could now be mapped with rule (5.2) to an allocentric location in direction $h_{i}$ in the reference system of the $\operatorname{sign} L_{\text {sign }}^{A}$ (the place the sign is pointing to). An example is presented in section 4.4.

Asking for an instruction from $L_{s}^{A}$ to $L_{d}^{A}$ is modeled in an equivalent way by the act of asking for directions (terminal $\operatorname{ask}\left[L_{s}^{A}\right]\left[L_{d}^{A}\right]$, rule (16)). The wayfinder may either ask another agent or a pedestrian navigation system. All communication processes lead back to the same instruction $I\left[L_{s}^{A}\right]\left[L_{d}^{A}\right]$ which may now be partially processable using the new route knowledge. We illustrate this with an example in subsection 4.5 .

\section{Wayfinding Scenarios}

In this section, we illustrate with examples how different types of wayfinding behavior can be modeled using our wayfinding grammar. 


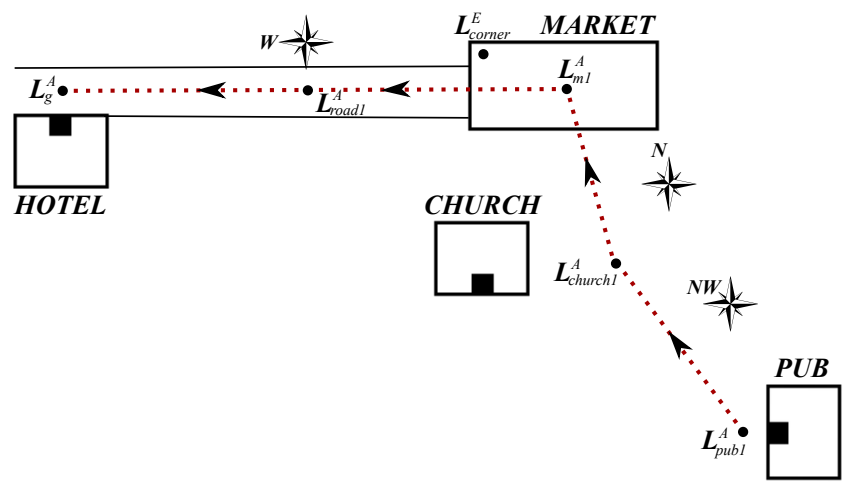

Fig. 3: Example wayfinding scenario.

Suppose the following scenario: Susi is visiting a foreign city of which she has so far acquired only limited spatial knowledge. She has just left the pub and is now trying to find her way back to the hotel (See Fig. 3).

\subsection{Following a Path from Internal Memory}

In the first example, we assume Susi remembers the path from previous experience, i.e., her accessibility graph is initialized as follows

$$
\begin{array}{lll}
A c c= & \left(L_{\text {pub } 1}^{A}, L_{\text {church } 1}^{A}, h_{N W}\right), & \left(L_{\text {church } 1}^{A}, L_{m 1}^{A}, h_{N}\right) \\
& \left.\left(L_{m 1}^{A}, L_{\text {road1 } 1}^{A}, h_{W}\right), \quad\left(L_{\text {road1 } 1}^{A}, L_{g}^{A}, h_{W}\right)\right) \\
\text { with } & L_{\text {pub1 } 1}^{A}=\left(A_{\text {pub }}, \text { rel } l_{\text {infront }}\right), & L_{\text {church } 1}^{A}=\left(A_{\text {church }}, \text { rel }_{\text {left }}\right), \\
& L_{m 1}^{A}=\left(A_{\text {market }}, \text { rel }_{\text {on }}\right), & L_{\text {road } 1}^{A}=\left(A_{\text {mainroad }},\right. \text { rel } \\
& L_{g}^{A}=\left(A_{\text {hotel }}, \text { rel }_{\text {infront }}\right) &
\end{array}
$$

Susi particularly remembers the market as a central place in this city. Thus, even though the accessibility graph is complete, she applies a planning strategy by structuring her plan into two sub-plans (pub to market, market to goal). The sub-plans are converted into instruction sequences which are sequentially turned into allocentric locations. Since her route memory is reliable, these allocentric locations can successfully be transformed into egocentric locations towards which she locomotes.

Figure 4 illustrates the production tree after applying rules in the following order: (10), (7), (1), (2), (3), (5.2), (6), (12), (5.1), (6), (12). The resulting termi-

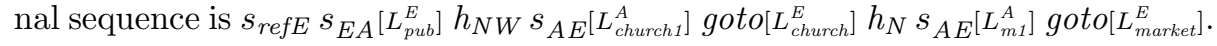
The exactly same procedure could now be applied to the second sub-plan $P\left[L_{m 1}^{A}\right]\left[L_{g}^{A}\right]$ which would lead Susi to her goal. 


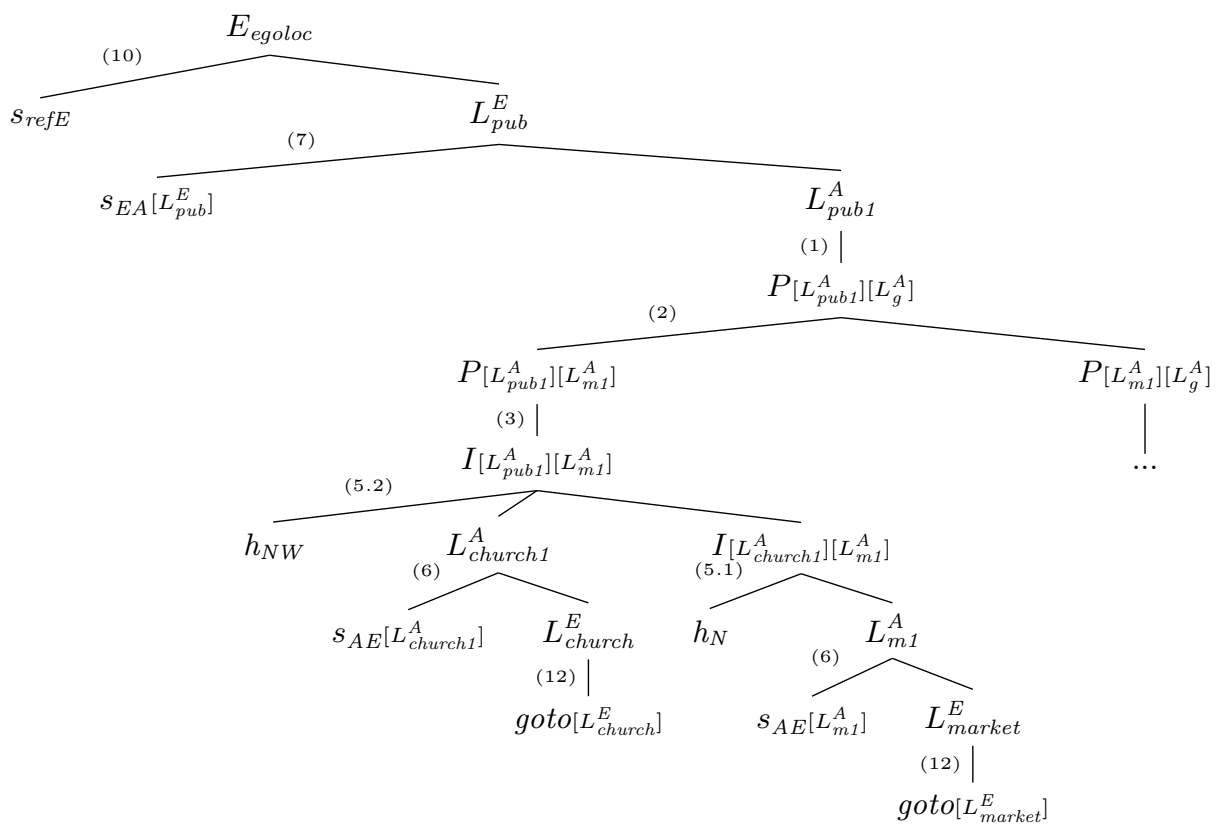

Fig. 4: An example production tree for wayfinding based on complete background knowledge with sub-planning (example in section 4.1).

\subsection{Explorative Path Search}

Let us now look at Susi's remaining path from the market to the hotel. In the second example, Susi again has complete knowledge on accessibility, but a construction fence obscures the transition from the market to the main road. Thus, the rule transforming the allocentric location $L_{\text {road1 }}^{A}$ into an egocentric location is not available in the rule set (the rule set was updated in $f_{\text {Perc }}$ when Susi entered the market with goto[ $\left.L_{\text {market }}^{E}\right]$, see Table 2 ).

Susi decides to explore her environment to find a way around the construction fence. She stays in direction $h_{W}$ and, with a visual search $\left(s_{\text {exp }}\right)$, identifies one promising egocentric location at the corner of the market $\left(L_{\text {corner }}^{E}\right)$ from which she thinks the road might be accessible. She locomotes to $L_{\text {corner }}^{E}$ and, indeed, from this new location she is able to find an egocentric location in her field of view that corresponds to $L_{\text {road } 1}^{A}$. Figure 5 illustrates how explorative path search appears in the production tree. Note that rule (14) can be applied multiple times to yield a longer search process.

\subsection{Map-Based Wayfinding}

In this example, Susi only knows the way from the pub to the market, but not how to get from the market to the hotel, i.e., $\left(L_{m 1}^{A}, L_{\text {road1 }}^{A}, h_{W}\right)$ and $\left(L_{\text {road1 }}^{A}, L_{g}^{A}\right.$, $\left.h_{W}\right)$ are missing in Acc. 


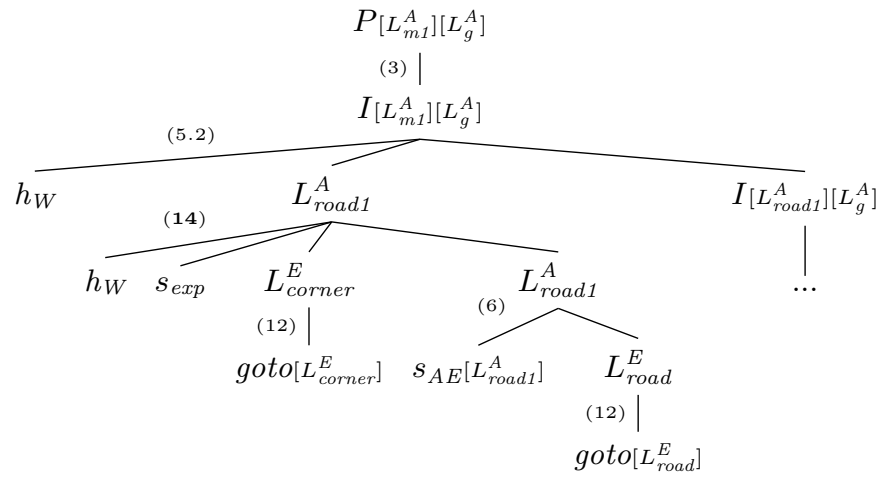

Fig. 5: Partial production tree for wayfinding with explorative path search (example in section 4.2).

Fortunately, Susi finds a public you-are-here map at the market which will help her updating her accessibility graph. First, she transforms her current allocentric location to a location in the map reference system (i.e., she localizes herself on the map). Then she successively moves her visual attention on the map towards West, detecting two survey locations $L_{\text {road }}^{S}$ and $L_{\text {hotel }}^{S}$ which are acessible from her location. She notices that the second, $L_{\text {hotel }}^{S}$, corresponds to her allocentric goal location $L_{g}^{A}$. Thus, her accessibility graph is completed, and she stops using the map. Figure 6 illustrates the according production tree.

\subsection{Use of Signage}

As in section 4.3, Susi does not know the way from the market to the hotel. This time she uses a sign with the name of her hotel pointing towards West. Reading the sign $\left(s_{s i g n}\right)$ enables her to update the accessibility graph Acc (with function $f_{\text {RemC }}$, See Table 2). She now knows that she can go from the market to the road $\left(\left(L_{m 1}^{A}, L_{\text {road1 }}^{A}, h_{W}\right) \in A c c\right)$, and that she can create an instruction from this (function $\left.f_{\text {Instr }}\right)^{5}$. Thus, she now heads towards West, searches for the allocentric location $L_{\text {road1 }}^{A}$, matches it to an egocentric location, and starts locomoting. Figure 7 (left) illustrates this example.

\subsection{Asking for Directions}

Again, Susi has no previous knowledge on the second part of her journey. She asks a passersby for directions from the market to her hotel $\left(\operatorname{ask}\left[L_{m 1}^{A}\right]\left[L_{g}^{A}\right]\right)$. The answers update her accessibility graph, and she is now able to follow these instructions to the goal (see Figure 7, right).

\footnotetext{
${ }^{5}$ A complex sign can add more than one edge to Acc.
} 


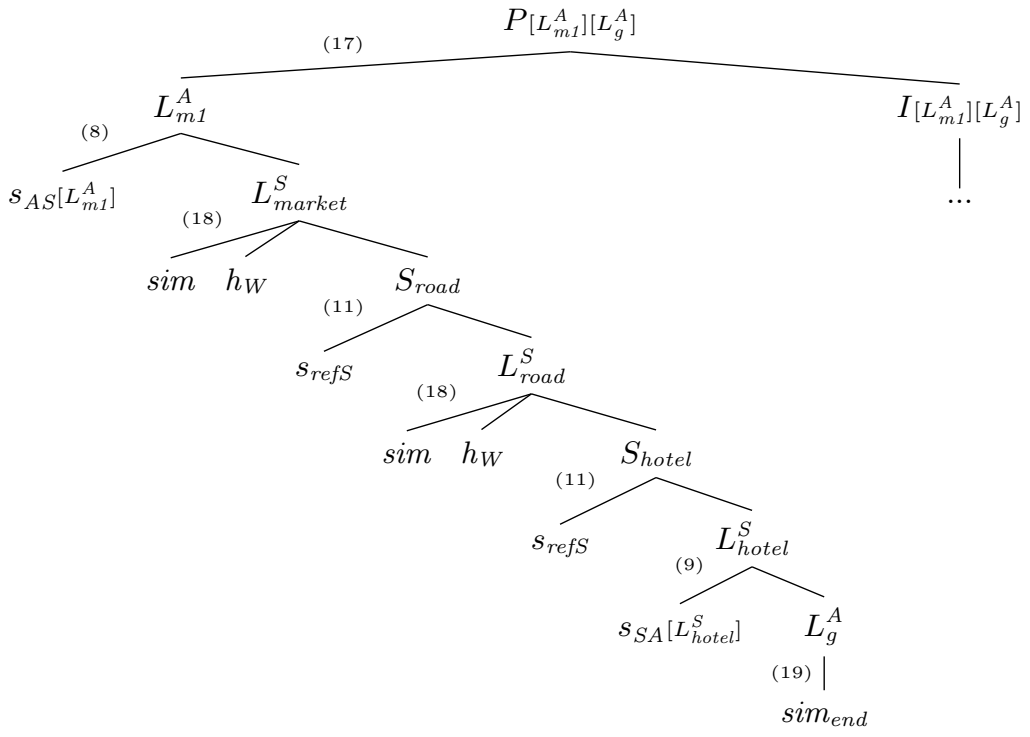

Fig. 6: Partial production tree for wayfinding with a map (example in section 4.3).
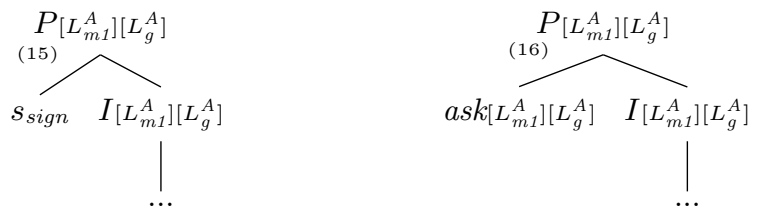

Fig. 7: Partial production trees for wayfinding with signage (left, see section 4.4) and with asking for directions (right, see section 4.5).

\section{Discussion and Outlook}

We argued that, in contrast to wayfinding decision models based on conditionaction rules (cf. Section 1), our approach does not make claims on how people actually make wayfinding decisions and how they search for locations and objects. The open slots in our model correspond precisely to these knowledge gaps and are simply the procedures necessary to generate the language defined by our grammar (cf. Table 2). The flexibility we gain is that we now have a precise model that separates "what we know" from "what we do not yet know".

In addition, we can now test our model together with particular (ad-hoc) procedures which might fill these slots based on embedding them in wayfinding simulations and comparing results with empirical observations. Testing could be achieved in three steps. First, the wayfinding behaviors defined in Definition 3 need to be tracked, e.g., using mobile eye tracking technology, as well as sensors such as GPS, accelerometer, and compass. Second, the collected data need to be 
processed and analyzed in order to match the actions to wayfinding behaviors. For visual search behaviors we propose to learn classifiers trained with machine learning from empirically collected data [18]. Third, the production trees need to be generated and compared against the empirical data. A parser (e.g., a modified version of an Earley Parser [4]) could be developed which produces all trees that are possible given the recognized behaviors and finally, the most likely parse tree could be selected using a selection algorithm and a probabilistic model. The probabilities of certain productions will depend on the complexity of a particular wayfinding situation [6], i.e., a model of the environment, the user, and the instruction complexity. Another challenge here, as in most approaches based on formal grammars, consists in ambiguities: sometimes there will be more than one possible parse tree that explains a given behavior/terminal sequence. Quantifying the amount of this ambiguity in real wayfinding situations is one opportunity for future research.

Even though our proposed model is amodal, processes as terminals (See Definition 3) might obviously be translated into visual behavior. However, this ignores that people also use auditory and haptic senses during wayfinding. For instance, a ringing church bell can be used to localize a church in an ego-centric reference system, while haptic maps enable non-visual search in a survey system [28]. Loomis et al. [29] argue that, instead of using a visual image, humans convert all types of sensory input to a spatial image, i.e., an amodal representation kept in working memory that abstracts from the sensor(s) from which it has been derived. A possible way to model different modalities in our grammar could be to replace the 'search' terminals $\left(s_{x}\right)$ by according Sense $_{x}$ non-terminals. Further production rules (called 'convert sensory input to spatial image') would map these Sense $_{x}$ non-terminals to either one or a sequence of terminals for auditory, visual, and haptic sensing. Finding sensoric interpretations for the wayfinding actions in our model and integrating them over different perceptual modalities is a topic for future research.

Using a computational formalism to simulate the wayfinding process can be useful, keeping in mind that a cognitive process is organized very differently from an algorithm in a computing machine. This leads to some general open methodical questions. First, grammars are not the only formalism that can be used for modeling wayfinding. One specific advantage of grammars compared to other rule-based systems and workflow engines, is that their expressiveness as well as computational complexity of parsing are well-understood. Second, cognitive and brain processes are typically highly parallel, i.e., different spatial representations are computed simultaneously [40], while our grammar execution is modeled as a single process. For example, we decided to represent route knowledge primarily in terms of allocentric reference systems, while in cognition, it may be stored in both egocentric and allocentric form [31]. Second, while our grammar in principle covers different kinds of externalized and internalized knowledge sources, it currently does not distinguish different kinds of systems of the same reference type, for example, different maps for indoor and outdoor that involve different sensed behaviors. 
Furthermore, our model should be enriched by further aspects of the wayfinding process. This includes, i.a., modeling the continuous modification of the perceptual array during movement, getting lost, aspects of aided and unaided wayfinding [45], the knowledge exchange between people (cf. $[44,43,10]$ ), and the interaction of people with artifacts.

\section{References}

1. Anderson, J.R., Matessa, M., Lebiere, C.: ACT-R: A Theory of Higher Level Cognition and Its Relation to Visual Attention. Human-Computer Interaction 12(4), 439-462 (Dec 1997)

2. Arthur, P., Passini, R.: How the wayfinding process works. In: Wayfinding: people, signs, and architecture, pp. 26-39. McGraw-Hill, New York, USA (1992)

3. Downs, R.M., Stea, D.: The world in the head. In: Maps in Minds: Reflections on Cognitive Mapping, chap. 4, pp. 125-135. Harper \& Row Series in Geography, Harper \& Row (1977)

4. Earley, J.: An efficient context-free parsing algorithm. Communications of the ACM 13, 94-102 (1970)

5. Frank, A.U.: Formal Models for Cognition. Taxonomy of Spatial Location Description and Frames of Reference. In: Freksa, C., Habel, C., Wender, K. (eds.) Spatial Cognition, Lecture Notes in Computer Science, vol. 1404, pp. 293-312. Springer Berlin Heidelberg (1998)

6. Giannopoulos, I., Kiefer, P., Raubal, M., Richter, K.F., Thrash, T.: Wayfinding Decision Situations: A Conceptual Model and Evaluation. In: Proceedings of the Eighth International Conference on Geographic Information Science (GIScience 2014), pp. 221-234. Springer International Publishing (2014)

7. Golledge, R.G.: Place recognition and wayfinding: Making sense of space. Geoforum 23, 199-214 (1992)

8. Golledge, R.G.: Human Wayfinding and Cognitive Maps. In: Wayfinding Behavior: Cognitive Mapping and Other Spatial Processes, chap. 1, pp. 5-45. The Johns Hopkins University Press (1999)

9. Gopal, S., Klatzky, R.L., Smith, T.R.: Navigator: A psychologically based model of environmental learning through navigation. Journal of Environmental Psychology 9(4), 309-331 (Dec 1989)

10. Hahn, J., Weiser, P.: A quantum formalization for communication coordination problems. In: Quantum Interaction, 8th International Conference, Lecture Notes in Computer Science. vol. 8951, p. 7. Springer (2015)

11. Haken, H., Portugali, J.: Synergetics, inter-representation networks and cognitive maps. In: The construction of cognitive maps, pp. 45-67. Springer (1996)

12. Hutchins, E.: Distributed cognition. International Encyclopedia of the Social and Behavioral Sciences. Elsevier Science (2000)

13. Hutchins, E.L.: Cognition in the wild. MIT press, 2 edn. (1996)

14. Kesner, R.P., Creem-Regehr, S.H.: Parietal contributions to spatial cognition. In: Handbook of spatial cognition, pp. 35-63. American Psychological Association (2013)

15. Kiefer, P.: Spatially constrained grammars for mobile intention recognition. In: Spatial Cognition VI. Learning, Reasoning, and Talking about Space, pp. 361377. Springer (2008)

16. Kiefer, P.: Mobile Intention Recognition. Springer, New York (2011) 
17. Kiefer, P., Giannopoulos, I.: Gaze map matching: Mapping eye tracking data to geographic vector features. In: Proceedings of the 20th SIGSPATIAL International Conference on Advances in Geographic Information Systems. pp. 359-368. ACM, New York, NY, USA (2012)

18. Kiefer, P., Giannopoulos, I., Raubal, M.: Using Eye Movements to Recognize Activities on Cartographic Maps. In: Proceedings of the 21st SIGSPATIAL International Conference on Advances in Geographic Information Systems. pp. 498-501. ACM, New York, NY, USA (2013)

19. Kiefer, P., Giannopoulos, I., Raubal, M.: Where am I? Investigating map matching during self-localization with mobile eye tracking in an urban environment. Transactions in GIS 18(5), 660-686 (2014)

20. Kitchin, R.M.: Cognitive maps: What are they and why study them? Journal of environmental psychology 14(1), 1-19 (1994)

21. Klippel, A., Tappe, H., Kulik, L., Lee, P.U.: Wayfinding choremes - A language for modeling conceptual route knowledge. Journal of Visual Languages and Computing 16(4), 311-329 (2005)

22. Knuth, D.E.: Semantics of context-free languages. Mathematical Systems Theory 2, 127-145 (1968)

23. Kuhn, W.: Semantic reference systems. International Journal of Geographical Information Science 17(5), 405-409 (2003)

24. Kuipers, B.: Modeling Spatial Knowledge. Cognitive Science 2(2), 129-153 (1978)

25. Leiser, D., Zilbershatz, A.: The Traveller: A Computational Model of Spatial Network Learning. Environment and Behavior 21(4), 435-463 (1989)

26. Levinson, S.C.: Space in language and cognition: Explorations in cognitive diversity, vol. 5. Cambridge University Press (2003)

27. Logan, G.D., Sadler, D.D.: A computational analysis of the apprehension of spatial relations. In: Language and space. Language, speech, and communication, pp. 493529. MIT Press (1996)

28. Lohmann, K., Eschenbach, C., Habel, C.: Linking spatial haptic perception to linguistic representations: assisting utterances for tactile-map explorations. In: Spatial information theory, pp. 328-349. Springer (2011)

29. Loomis, J.M., Klatzky, R.L., Giudice, N.A.: Representing 3d space in working memory: Spatial images from vision, hearing, touch, and language. In: Multisensory imagery, pp. 131-155. Springer (2013)

30. MacEachren, A.M.: How maps work: representation, visualization, and design. New York: Guilford Press (1995)

31. McNamara, T.P.: Spatial memory: properties and organization. In: Handbook of spatial cognition, pp. 173-190. American Psychological Association (2013)

32. Montello, D.R.: Navigation. In: Cambridge handbook of visuospatial thinking., pp. 257-294. Cambridge University Press (2005)

33. Montello, D.R.: Scale and multiple psychologies of space. In: Spatial information theory a theoretical basis for GIS, pp. 312-321. Springer (1993)

34. Passini, R.: Wayfinding: A conceptual framework. Urban Ecology 5(1), 17-31 (Apr 1981)

35. Raubal, M., Worboys, M.: A Formal Model of the Process of Wayfinding in Built Environments. In: Freksa, C., Mark, D. (eds.) Spatial Information Theory. Cognitive and Computational Foundations of Geographic Information Science, LNCS, vol. 1661, pp. 381-399. Springer Berlin Heidelberg (1999)

36. Richter, K.F., Winter, S.: Landmarks. Springer (2014)

37. Scheider, S.: Grounding geographic information in perceptual operations, Frontiers in Artifical Intelligence and Applications, vol. 244. IOS Press (2012) 
38. Schlieder, C.: Representing the meaning of spatial behavior by spatially grounded intentional systems. In: GeoSpatial Semantics, pp. 30-44. Springer (2005)

39. Shelton, A.L., McNamara, T.P.: Systems of spatial reference in human memory. Cognitive psychology 43(4), 274-310 (2001)

40. Simmering, V.R., Schutte, A.R., Spencer, J.P.: Generalizing the dynamic field theory of spatial cognition across real and developmental time scales. Brain research 1202, 68-86 (2008)

41. Smith, T.R., Pellegrino, J.W., Golledge, R.G.: Computational process modeling of spatial cognition and behavior. Geographical Analysis 14(4), 305-325 (1982)

42. Tversky, B.: Cognitive maps, cognitive collages, and spatial mental models. In: Frank, A., Campari, I. (eds.) Spatial Information Theory A Theoretical Basis for GIS, LNCS, vol. 716, pp. 14-24. Springer Berlin Heidelberg (1993)

43. Weiser, P.: A Pragmatic Communication Model for Way-finding Instructions. Ph.D. thesis, Vienna University of Technology. Department of Geodesy and Geoinformation. Research Group Geoinformation. (2014)

44. Weiser, P., Frank, A.U.: Cognitive Transactions - A Communication Model. In: Conference on Spatial Information Theory (COSIT), number 8116. pp. 129-148. Springer International Publishing (2013)

45. Wiener, J.M., Büchner, S.J., Hölscher, C.: Taxonomy of human wayfinding tasks: A knowledge-based approach. Spatial Cognition \& Computation 9(2), 152-165 (2009) 